\title{
Marshall-Olkin Discrete Uniform Distribution
}

\author{
E. Sandhya ${ }^{1}$ and C. B. Prasanth ${ }^{2}$ \\ ${ }^{1}$ Department of Statistics, Prajyothi Niketan College, Pudukkad, Thrissur-680 301, India \\ ${ }^{2}$ Department of Statistics, St. Thomas College, Pala, 686574, India
}

Correspondence should be addressed to C. B. Prasanth; cbpwarrier@yahoo.co.in

Received 24 November 2013; Revised 11 February 2014; Accepted 20 February 2014; Published 7 April 2014

Academic Editor: Serkan Eryílmaz

Copyright (C) 2014 E. Sandhya and C. B. Prasanth. This is an open access article distributed under the Creative Commons Attribution License, which permits unrestricted use, distribution, and reproduction in any medium, provided the original work is properly cited.

\begin{abstract}
We introduce and characterize a new family of distributions, Marshall-Olkin discrete uniform distribution. The natures of hazard rate, entropy, and distribution of minimum of sequence of i.i.d. random variables are derived. First order autoregressive (AR (1)) model with this distribution for marginals is considered. The maximum likelihood estimates for the parameters are found out. Also, the goodness of the distribution is tested with real data.
\end{abstract}

\section{Introduction}

Marshall and Olkin [1] introduced a new method for adding a parameter to a family of distributions with application to the exponential and Weibull families. Jose and Alice [2] discussed Marshall-Olkin family of distributions and their applications in time series modeling and reliability. Jose and Krishna [3] have developed Marshall-Olkin extended uniform distribution. These works and most of the references there in, deal with continuous distribution. Not much work is seen in the discrete case. The reason behind this may be that it is difficult to obtain compact mathematical expressions for moments, reliability, and estimation in the discrete set up.

If $\bar{F}(x)$ is the survival function of a distribution, then, by Marshall-Olkin method, we get another survival function $\bar{G}(x)$, by adding a new parameter $\theta$ to it. That is,

$$
\bar{G}(x, \theta)=\frac{\theta \bar{F}(x)}{(1-(1-\theta) \bar{F}(x))}, \quad-\infty<x<\infty, \theta>0 .
$$

Then the corresponding distribution function is $G(x, \theta)=1-$ $\bar{G}(x, \theta)=F(x) /(1-(1-\theta) \bar{F}(x))$.
Now its probability mass function (p.m.f.) is

$$
\begin{aligned}
g(x, \theta) & =G(x, \theta)-G(x-1, \theta) \\
& =\frac{\theta f(x)}{[1-(1-\theta) \bar{F}(x)][1-(1-\theta) \bar{F}(x-1)]},
\end{aligned}
$$

where $f(x)$ is the p.m.f. corresponding to $F(x)$.

The hazard rate of $X$ is

$$
\gamma_{G}(x)=\frac{g(x)}{\bar{G}(x)}
$$

We consider a new family of distributions by adding an additional parameter by using the Marshall-Olkin scheme (Marshall and Olkin [1] in Section 2). The nature of hazard rate, entropy and expectation are derived. In the third section, distributions of minimum sequence of i.i.d. random variables are found out. An AR (1) model with new Marshall-Olkin discrete uniform distribution is discussed in the next section. In the fifth section, the maximum likelihood estimate (m.l.e.) for the parameters is found out and, in the last section, the goodness of the distribution is tested with a real life data. 

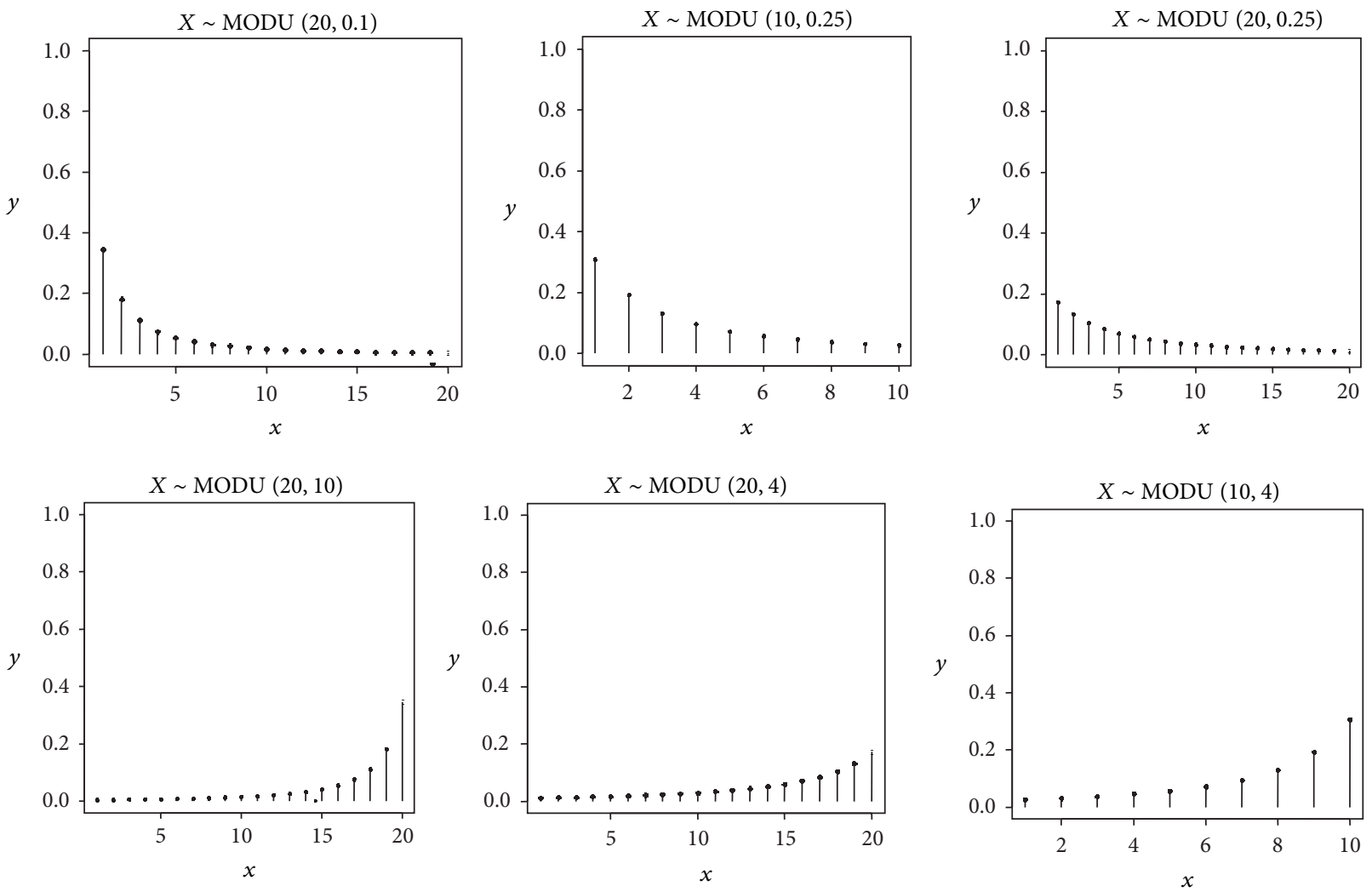

Figure 1: P.m.f. of $X \sim$ MODU.

\section{Marshall-Olkin Discrete Uniform Distribution}

Let $X$ be a discrete random variable following uniform distribution with p.m.f. $f(x)=1 / a, x=1,2,3, \ldots, a$. We have the distribution function $F(x)=x / a$ and the survival function $\bar{F}(x)=(a-x) / a$. By Marshall-Olkin method, we can form another survival function of Marshall-Olkin discrete uniform (MODU) distribution by substituting $\bar{F}(x)=(a-$ $x) / a$ in $\bar{G}(x, \theta)$, in (1), and we get

$$
\bar{G}(x, \theta)=\frac{\theta(a-x)}{[a \theta+(1-\theta) x]}, \quad 0<\theta<\infty .
$$

We write $X \sim \operatorname{MODU}(a, \theta)$ for a random variable (r.v.) with $\bar{G}(x, \theta)$ given in (4).

2.1. Stability Property of the New Family. If we apply the same method again into the new family, by adding a new parameter " $\alpha$ " $(\alpha>0)$ to the reliability function, we get $\bar{G}_{1}(x)=(\alpha \theta)(a-$ $x) /[a-(1-\alpha \theta)(a-x)]$, which is the reliability function of the new Marshall-Olkin family with parameters $(a, \alpha \theta)$.

2.2. Probability Mass Function. From (2), consider the probability mass function p.m.f.

$$
g(x, \theta)=\frac{\theta f(x)}{[1-(1-\theta) \bar{F}(x)][1-(1-\theta) \bar{F}(x-1)]},
$$

where $f(x)$ is the p.m.f. corresponding to $F(x)$. That is,

$$
g(x, \theta)=\frac{a \theta}{\{[a \theta+(1-\theta) x][a \theta+(1-\theta)(x-1)]\}} .
$$

Now we plot the p.m.f. of $X \sim$ MODU for different values of $a$ and $\theta$ (Figure 1).

Remark 1. If $X$ follows $\operatorname{MODU}(a, \theta)$, then $g(x, a, \theta)=g(a-$ $x+1, a, 1 / \theta), x=1,2, \ldots, a, \theta>0$.

Now, our next interest is to check whether (6) characterizes MODU. If $X$ satisfies the condition $g(x, a, \theta)=g(a-$ $x+1, a, 1 / \theta), x=1,2, \ldots, a, \theta>0$, this should be true for $\theta=1$ also. For $\theta=1$, it gives the parent distribution itself before reparameterization. Thus, Remark 1 implies that $P(x)=P(a-x+1), x=1,2,3, \ldots, a$, where $P(x)$ is the p.m.f. of discrete uniform distribution with parameter " $a$ ". That is, $P(1)=P(a), P(2)=P(a-1), \ldots, P(a)=P(1)$. This relation need not characterize a discrete uniform distribution, when $a>2$. For example, let $a=3$; then we get $P(1)=P(3)$. Since $P(1)+P(2)+P(3)=1$, we get $P(2)=1-P(1)-P(3)$. That is $P(2)=1-2 P(1)$. Let $P(1)=0.1=P(3)$ imply $P(2)=0.8$, which is not discrete uniform. Thus we have the following.

Theorem 2. A random variable $X$ follows $\operatorname{MODU}(2, \theta), x=$ 1,2 if and only if its p.m.f. satisfies

$$
g(x, a, \theta)=g\left(a-x+1, a, \frac{1}{\theta}\right) .
$$


TABLe 1: Expectation and standard deviation (sd) with different " $a$ " and " $\theta$ " $(\theta<1)$ for $X \sim \operatorname{MODU}(a, \theta)$.

\begin{tabular}{|c|c|c|c|c|c|c|c|c|}
\hline \multirow{2}{*}{$a$} & \multicolumn{2}{|c|}{$\theta=0.133$} & \multicolumn{2}{|c|}{$\theta=0.25$} & \multicolumn{2}{|c|}{$\theta=0.5$} & \multicolumn{2}{|c|}{$\theta=0.75$} \\
\hline & $E(X)$ & $\operatorname{sd}(X)$ & $E(X)$ & $\operatorname{sd}(X)$ & $E(X)$ & $\operatorname{sd}(X)$ & $E(X)$ & $\operatorname{sd}(X)$ \\
\hline 10 & 2.597426 & 2.186733 & 3.358938 & 2.519965 & 4.375428 & 2.778938 & 5.026709 & 2.855936 \\
\hline 12 & 2.995745 & 2.638189 & 3.919444 & 3.033456 & 5.145940 & 3.341060 & 5.930269 & 3.432611 \\
\hline 15 & 3.597637 & 3.312588 & 4.762709 & 3.801608 & 6.302744 & 4.182802 & 7.286014 & 4.296371 \\
\hline 20 & 4.606958 & 4.43258 & 6.171538 & 5.079024 & 8.232135 & 5.583787 & 9.546128 & 5.734303 \\
\hline 25 & 5.620115 & 5.550024 & 7.582418 & 6.354713 & 10.16236 & 6.983619 & 11.80657 & 7.171237 \\
\hline 30 & 6.635235 & 6.666144 & 8.994330 & 7.629531 & 12.09300 & 8.382876 & 14.06717 & 8.607673 \\
\hline 50 & 10.70370 & 11.12519 & 14.64612 & 12.72530 & 19.81722 & 13.97760 & 23.11022 & 14.35143 \\
\hline 75 & 15.79535 & 16.69483 & 21.71398 & 19.09236 & 29.47374 & 20.96928 & 34.41451 & 21.52963 \\
\hline 100 & 20.88903 & 22.26307 & 28.78287 & 25.45854 & 39.13069 & 27.96038 & 45.71897 & 28.70733 \\
\hline
\end{tabular}

Proof. Suppose that $g(x, a, \theta)=g(a-x+1, a, 1 / \theta)$ is true for Marshall-Olkin (MO) scheme, then its parent distribution is discrete uniform on $\{1,2\}$. That is,

$$
g(x, 2, \theta)=g\left(2-x+1,2, \frac{1}{\theta}\right)=\frac{1}{2},
$$

that is,

$$
g(1,2, \theta)=g\left(2,2, \frac{1}{\theta}\right)
$$

We know the p.m.f. $g(x, a, \theta)=\theta f(x) /[1-(1-\theta) \bar{F}(x-1)][1-$ $(1-\theta) \bar{F}(x)]$. That is,

$$
\begin{aligned}
g(1,2, \theta) & =\frac{\theta P(1)}{[1-(1-\theta) \bar{F}(0)][1-(1-\theta) \bar{F}(1)]} \\
& =\frac{\theta P(1)}{[1-(1-\theta)][1-(1-\theta) \bar{F}(1)]}=\frac{1}{2},
\end{aligned}
$$

by (8); that is,

$$
\begin{aligned}
2 \theta P & (1)=[1-(1-\theta)][1-(1-\theta) \bar{F}(1)] \\
g\left(2,2, \frac{1}{\theta}\right) & =\frac{P(2)}{\theta[1-(1-1 / \theta) \bar{F}(1)][1-(1-1 / \theta) \bar{F}(2)]} \\
& =\frac{P(2)}{\theta[1-(1-1 / \theta) \bar{F}(1)]} \\
& =\frac{P(2)}{\theta[1-((\theta-1) / \theta) \bar{F}(1)]} .
\end{aligned}
$$

That is, $g(2,2,1 / \theta)=P(2) /[\theta-(\theta-1) \bar{F}(1)]=1 / 2$, by $(8)$.

That is, $2 P(2)=[\theta-(\theta-1) \bar{F}(1)]$ or $2 P(2)-\theta=-(\theta-$ 1) $\bar{F}(1)$. We get,

$$
\bar{F}(1)=\frac{(2 P(2)-\theta)}{1-\theta} .
$$

By substituting this in (11),

$$
\begin{aligned}
2 \theta P(1)= & {[1-(1-\theta)]\left[1-(1-\theta)\left(\frac{(2 P(2)-\theta)}{(1-\theta)}\right)\right] } \\
= & {[1-(1-\theta)][1-(2 P(2)-\theta)], } \\
& 2 \theta P(1)=\theta[1-(2 P(2)-\theta)] .
\end{aligned}
$$

That is, $2 P(1)=1-2 P(2)+\theta$ or $2(P(1)+P(2))=1+\theta$. So $2=1+\theta$, since $P(1)+P(2)=1$.

That is, $\theta=1$. But, when $\theta=1$, it corresponds to discrete uniform distribution on $\{1,2\}$. The converse is straight forward.

Remark 3. It is clear that MODU distribution does not possess additive property.

Remark 4. MODU is not infinitely divisible (i.d.), since its support is on $\{1,2,3, \ldots, a\}$, a finite range. It is not log convex, since the class of log convex distribution forms a subclass of the class of i.d. distributions.

Corollary 5. If $X \sim \operatorname{MODU}(a, \theta)$, then $\bar{F}(x) \leq \bar{G}(x, \theta) \leq$ $\theta \bar{F}(x)$, if $\theta \leq 1$, and $\theta \bar{F}(x)<\bar{G}(x, \theta)<\bar{F}(x)$, if $0<\theta<1$.

Proof. Let $X \sim \operatorname{MODU}(a, \theta), \bar{F}(x)=(a-x) / a$ and $\bar{G}(x, \theta)=$ $\theta(a-x) /[a \theta+(1-\theta) x]$. So, $\bar{F}(x) \leq \bar{G}(x, \theta)$ implies that $(a-$ $x) / a \leq \theta(a-x) /[a \theta+(1-\theta) x]$. That is $1 / a-\theta /[a \theta+(1-\theta) x] \leq$ $0, a \theta+(1-\theta) x-a \theta \leq 0$, or $(1-\theta) x \leq 0$. This happens only when $\theta \geq 1$. Now assume that $\bar{G}(x, \theta) \leq \theta \bar{F}(x)$. That is, $\theta(a-x) /[a \theta+(1-\theta) x] \leq \theta(a-x) / a$. So $(1-\theta) a-(1-\theta) x \leq 0$; that is, $(a-x)(1-\theta) \leq 0$, which is true only when $\theta \geq 1$, since $(a-x) \geq 0$. Hence, the proof is completed.

2.3. Expectation, Standard Deviation, and Entropy of MODU Distribution. We numerically compute the expectation and standard deviation (Tables 1 and 2) and entropy (Table 3) of the MODU distribution with different $a$ and $\theta$, since compact 
TABLE 2: Expectation and standard deviation with different " $a$ " and " $\theta$ " $(\theta>1)$ for $X \sim \operatorname{MODU}(a, \theta)$.

\begin{tabular}{lcccccccc}
\hline$a$ & \multicolumn{2}{c}{$\theta=1.33$} & \multicolumn{2}{c}{$\theta=2$} & \multicolumn{2}{c}{$\theta=4$} & \multicolumn{2}{c}{$\theta=7.5$} \\
& $E(X)$ & $\operatorname{sd}(X)$ & $E(x)$ & $\operatorname{sd}(x)$ & $E(x)$ & $\operatorname{sd}(x)$ & 2.51996 & 8.40257 \\
10 & 5.97329 & 2.855936 & 6.62457 & 2.77893 & 7.64106 & 2.18673 \\
12 & 7.06973 & 3.432611 & 7.85406 & 3.34106 & 9.08055 & 3.03345 & 10.0042 & 2.63818 \\
15 & 8.71398 & 4.296371 & 9.69725 & 4.18280 & 11.2372 & 3.80160 & 12.4023 & 3.31258 \\
20 & 11.4538 & 5.734303 & 12.7678 & 5.58378 & 14.8284 & 5.07902 & 16.3930 & 4.43258 \\
25 & 14.1934 & 7.171237 & 15.8376 & 6.98361 & 18.4175 & 6.35471 & 20.3798 & 5.55002 \\
30 & 16.9328 & 8.607673 & 18.9070 & 8.38287 & 22.0056 & 7.62953 & 24.3647 & 6.66614 \\
50 & 27.8897 & 14.35143 & 31.1827 & 13.9776 & 36.3538 & 12.7253 & 40.2963 & 11.1251 \\
75 & 41.5854 & 21.52963 & 46.5262 & 20.9692 & 54.2860 & 19.0923 & 60.2046 & 16.6948 \\
100 & 55.2810 & 28.70733 & 61.86931 & 27.96038 & 72.21713 & 25.45854 & 80.11097 & 22.26307 \\
\hline
\end{tabular}

TABle 3: Entropy with different " $a$ " and " $\theta$ " for $X \sim \operatorname{MODU}(a, \theta)$.

\begin{tabular}{lcccccccccc}
\hline$a$ & $\theta=0.1$ & $\theta=0.133$ & $\theta=0.25$ & $\theta=0.5$ & $\theta=0.75$ & $\theta=1.33$ & $\theta=2$ & $\theta=4$ & $\theta=7.5$ & $\theta=10$ \\
\hline 10 & 1.5310 & 1.6923 & 1.9985 & 2.2241 & 2.2889 & 2.2889 & 2.2241 & 1.9985 & 1.6923 & 1.5310 \\
15 & 1.9142 & 2.0846 & 2.4004 & 2.6290 & 2.6943 & 2.6943 & 2.6290 & 2.4004 & 2.0846 & 1.9142 \\
20 & 2.1933 & 2.3674 & 2.6868 & 2.9165 & 2.9819 & 2.9819 & 2.9165 & 2.6868 & 2.3674 & 2.1933 \\
30 & 2.5923 & 2.7692 & 3.0914 & 3.3218 & 3.3874 & 3.3874 & 3.3218 & 3.0914 & 2.7692 & 2.5923 \\
50 & 3.0997 & 3.2782 & 3.6017 & 3.8326 & 3.8982 & 3.8982 & 3.8326 & 3.6017 & 3.2782 & 3.0997 \\
100 & 3.7913 & 3.9705 & 4.2947 & 4.5257 & 4.5913 & 4.5913 & 4.5257 & 4.2947 & 3.9705 & 3.7913 \\
\hline
\end{tabular}

expressions are not available for calculating the same. We have

Shannon's Entropy

$$
\begin{aligned}
= & -\sum_{I} p\left(x_{i}\right) \log p\left(x_{i}\right), \quad i \in I=\{1,2, \ldots\} \\
= & -\sum \frac{a \theta}{\left[a \theta+(1-\theta) x_{i}\right]\left[a \theta+(1-\theta)\left(x_{i}-1\right)\right]} \\
& \times \log \frac{a \theta}{\left[a \theta+(1-\theta) x_{i}\right]\left[a \theta+(1-\theta)\left(x_{i}-1\right)\right]} .
\end{aligned}
$$

Remark 6. If $X \sim \operatorname{MODU}(a, \theta)$, then the $\operatorname{sd}(X)$ is decreasing with increasing value of $\theta$ when $\theta>1$ and the $\operatorname{sd}(X)$ increasing with increasing value of $\theta$ when $\theta<1$.

From Remark 1 and Tables 1 and 2, we have the following.

Remark 7. The $\operatorname{sd}(X)$ for $X \sim \operatorname{MODU}(a, \theta)$ is equal to $\operatorname{sd}(X)$ for $X \sim \operatorname{MODU}(a, 1 / \theta)$.

From Remark 1, Table 3, and Figure 2, the following is observed.

Remark 8. The entropy of $X \sim \operatorname{MODU}(a, \theta)$ is equal to the entropy of $X \sim \operatorname{MODU}(a, 1 / \theta)$.
TABLE 4: Mean, median, and mode of the MODU distribution with different $a$ and $\theta$.

\begin{tabular}{ccccc}
\hline$a$ & $\theta$ & Mean & Median & Mode \\
\hline \multirow{4}{*}{10} & 0.2 & 3.06 & 2 & 1 \\
& 0.5 & 4.40 & 3 & 1 \\
& 2 & 6.60 & 7 & 10 \\
& 5 & 7.90 & 9 & 10 \\
\hline \multirow{4}{*}{20} & 0.2 & 5.57 & 4 & 1 \\
& 0.5 & 8.23 & 7 & 1 \\
& 2 & 12.76 & 14 & 20 \\
& 5 & 15.40 & 17 & 20 \\
\hline \multirow{3}{*}{100} & 0.2 & 25.70 & 17 & 1 \\
& 0.5 & 39.10 & 34 & 1 \\
& 2 & 61.80 & 67 & 100 \\
& 5 & 75.20 & 84 & 100 \\
\hline
\end{tabular}

Let $X \sim \operatorname{MODU}(a, \theta)$; then the mean, median, and mode of the distribution (in Figure 1 and Tables 1 and 2) for different $a$ and $\theta$ are computed in Table 4 .

From Figure 1 and from Table 4, the following is clear.

Remark 9. The MODU distribution is positively skewed, when $\theta<1$, since mode $<$ median $<$ mean and the distribution is negatively skewed, when $\theta>1$, since mode $>$ median $>$ mean. Also, it is to be noted that the distribution is 

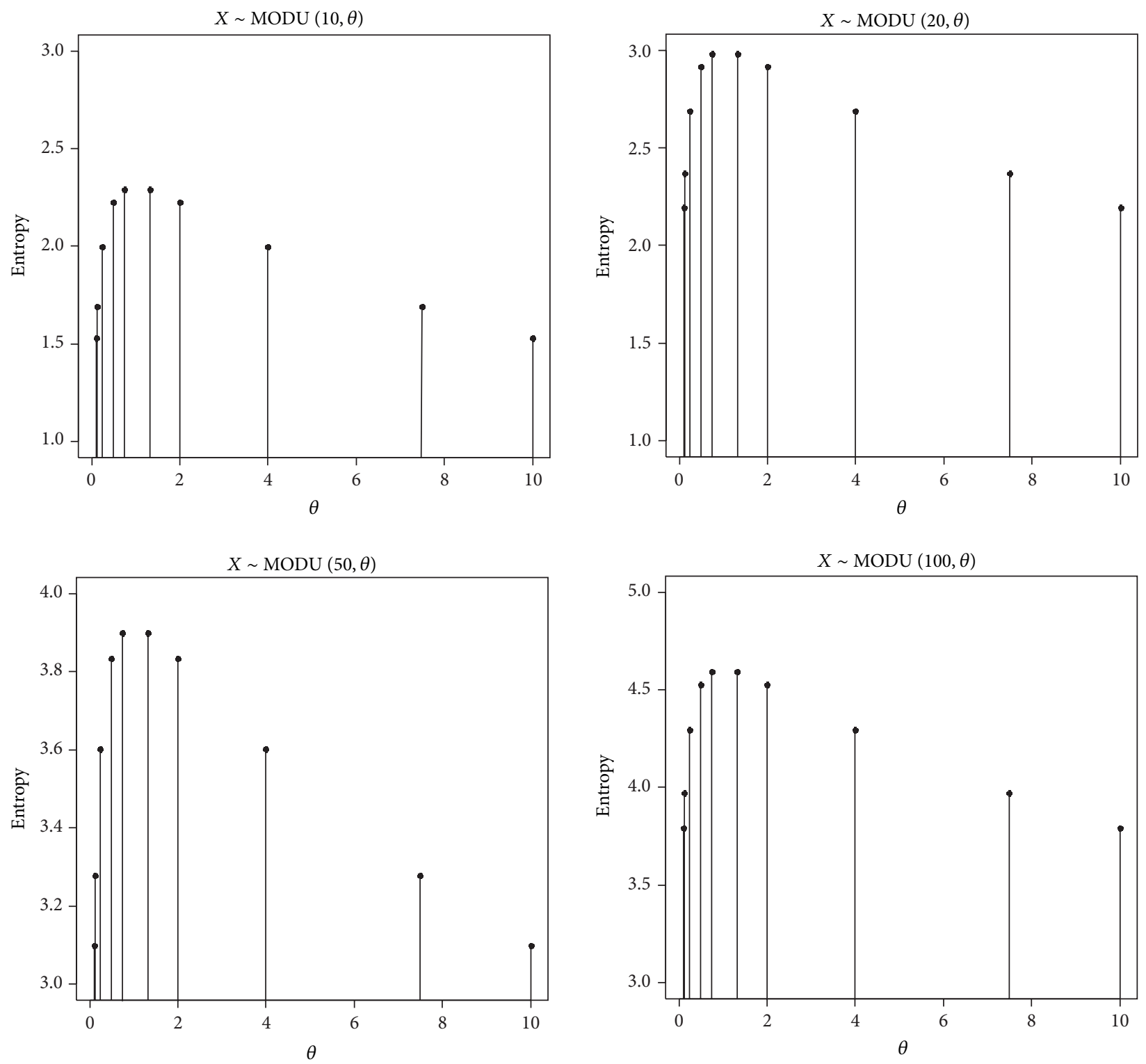

Figure 2: Entropy with different " $a$ " and “ $\theta$ " for $X \sim \operatorname{MODU}(a, \theta)$.

TABLE 5: Hazard function $\gamma_{G}(x)$ of $X \sim \operatorname{MODU}(a, \theta)$ with $a=10$.

\begin{tabular}{|c|c|c|c|c|c|c|c|}
\hline \multirow{2}{*}{$X$} & \multicolumn{7}{|c|}{$\theta$} \\
\hline & 0.75 & 0.5 & 0.2 & 0.1 & 2 & 5 & 7.5 \\
\hline 1 & 0.1481481 & 0.2222222 & 0.5555556 & 1.111111 & 0.05555556 & 0.02222222 & 0.01481481 \\
\hline 2 & 0.1612903 & 0.2272727 & 0.4464286 & 0.6578947 & 0.06578947 & 0.02717391 & 0.01824818 \\
\hline 3 & 0.1785714 & 0.2380952 & 0.3968254 & 0.5102041 & 0.07936508 & 0.03401361 & 0.02304147 \\
\hline 4 & 0.2020202 & 0.2564103 & 0.3787879 & 0.4504505 & 0.09803922 & 0.04385965 & 0.03003003 \\
\hline 5 & 0.2352941 & 0.2857143 & 0.3846154 & 0.4347826 & 0.1250000 & 0.05882353 & 0.04081633 \\
\hline 6 & 0.2857143 & 0.3333333 & 0.4166667 & 0.4545455 & 0.1666667 & 0.08333333 & 0.05882353 \\
\hline 7 & 0.3703704 & 0.4166667 & 0.4901961 & 0.5208333 & 0.2380952 & 0.1282051 & 0.09259259 \\
\hline 8 & 0.5405405 & 0.5882353 & 0.6578947 & 0.6849315 & 0.3846154 & 0.2272727 & 0.1694915 \\
\hline 9 & 1.052632 & 1.111111 & 1.190476 & 1.219512 & 0.8333333 & 0.5555556 & 0.4347826 \\
\hline
\end{tabular}


unimodal; that is, when $\theta<1$, the mode $=1$ and, when $\theta>1$, the mode $=a$.

Therefore, the MODU distribution can be applied for the data showing positive skewness when $\theta>1$ and the data showing negative skewness when $\theta<1$.

2.4. Hazard Function. The hazard function of $X \sim$ $\operatorname{MODU}(a, \theta)$ is

$$
\begin{aligned}
\gamma_{G}(x) & =\frac{g(x)}{\bar{G}(x)} \\
& =\frac{\theta(a-x+1) /[a \theta+(1-\theta)(x-1)]}{\theta(a-x) /[a \theta+(1-\theta) x]}-1 .
\end{aligned}
$$

That is,

$$
\gamma_{G}(x)=\frac{a}{(a-x)(a \theta+(1-\theta)(x-1))} .
$$

Theorem 10. Let $X \sim \operatorname{MODU}(a, \theta)$; then the distribution is with Increasing Failure Rate (IFR) when $\theta>(a-2 x) /(2 a-2 x)$, Decreasing Failure Rate (DFR) when $\theta<(a-2 x) /(2 a-2 x)$, and constant FR when $\theta=(a-2 x) /(2 a-2 x)$.

Proof. From (17), the hazard function of $X \sim \operatorname{MODU}(a, \theta)$ is $\gamma_{G}(x)=a /\{(a-x)(a \theta+(1-\theta)(x-1))\}$.

Then $\gamma_{G}(x+1)=a /(a-x-1)(a \theta+(1-\theta) x)$. If $\gamma_{G}(x+$ 1) $-\gamma_{G}(x)>0$, the distribution is with IFR.

That is, if $\gamma_{G}(x+1) / \gamma_{G}(x)>1$ or

$$
\begin{gathered}
\frac{(a-x)(a \theta+(1-\theta)(x-1))}{(a-x-1)(a \theta+(1-\theta) x)}>1, \\
(a-x)(a \theta+(1-\theta)(x-1)) \\
-(a-x-1)(a \theta+(1-\theta) x)>0, \\
(-a+2 a \theta+2 x-2 x \theta)>0, \\
\theta(2 a-2 x)>a-2 x \text { or }
\end{gathered}
$$

if $\theta>(a-2 x) /(2 a-2 x)$, the distribution is with IFR.

Similarly, if $\theta<(a-2 x) /(2 a-2 x)$, the distribution is with DFR, and if $\theta=(a-2 x) /(2 a-2 x)$, the distribution is with a constant FR.

We illustrate these results graphically (Figure 3) and numerically (Table 5).

From Figure 3, also, it is clear that the failure rate is IFR, when $\theta>(a-2 x) /(2 a-2 x)$, and DFR, when $\theta<(a-2 x) /(2 a-$ $2 x)$.

\section{MODU Distribution as the Distribution of Minimum of a Sequence of i.i.d. Random Variables}

The following theorem gives a characterization of minimum of a sequence of i.i.d. random variables following MODU distribution.
TABLE 6: Initial observations.

\begin{tabular}{lccccccc}
\hline & $N$ & $a$ & $\widehat{a}$ & $\theta$ & $\hat{\theta}$ & Mean $\hat{\theta}$ & $\mathrm{SE}(\widehat{\theta})$ \\
\hline 1 & & & & & 0.104118 & & \\
2 & & & & & 0.111474 & & \\
3 & & & & & 0.095902 & & \\
4 & & & & & 0.101358 & & \\
5 & & & & & 0.098971 & 0.1016693 & 0.1016693 \\
6 & 2500 & 20 & 20 & 0.1 & 0.095781 & & \\
7 & & & & & 0.101448 & & \\
8 & & & & & 0.098904 & & \\
9 & & & & & 0.103342 & & \\
10 & & & & & 0.105396 & & \\
\hline
\end{tabular}

Theorem 11. Let $\left\{X_{i}, i \geq 1\right\}$ be a sequence of i.i.d. random variables with common survival function $\bar{F}(x)$. Let $N$ be a geometric random variable independent of $\left\{X_{i}, i \geq 1\right\}$ such that $P(N=n)=p q^{n-1}, n=1,2,3, \ldots ; 0<p=\theta<1$, and $q=1-p$. Let $U_{N}=\min _{1 \leq i \leq n}\left(X_{i}\right)$. Then $\left\{U_{N}\right\}$ is distributed as MODU distribution if and only if $\left\{X_{i}\right\}$ follows discrete uniform distribution with $x=1,2,3, \ldots, a$.

Proof. Proof follows as in the same lines as given in Satheesh et al. [4-6] for a similar characterization of minimum of sequence of i.i.d. random variables.

The survival function of $U_{N}$ is $\bar{G}(x)=P\left(U_{N}>\right.$ $x)=\sum_{1}^{\infty}[\bar{F}(x)]^{n} P(N=n)=\sum_{1}^{\infty}[\bar{F}(x)]^{n} \theta(1 \quad-$ $\theta)^{n}=\theta \sum_{1}^{\infty}[\bar{F}(x)(1-\theta)]^{n}=\theta \bar{F}(x) /[1-(1-\theta) \bar{F}(x)]$. Substituting $\bar{F}(x)=(a-x) / a$, the survival function of the discrete uniform distribution; that is,

$$
\begin{aligned}
\bar{G}(x) & =\frac{\theta[(a-x) / a]}{[1-(1-\theta)((a-x) / a)]} \\
& =\frac{\theta(a-x)}{[a-(1-\theta)(a-x)]}=\frac{\theta(a-x)}{[a \theta+(1-\theta) x]},
\end{aligned}
$$

which is the survival function of $\operatorname{MODU}(a, \theta)$. By retracing the steps we can easily show the converse.

\section{AR (1) Model with MODU Distribution as Innovating Distribution}

Arnold and Robertson [7], Chernick [8], and Satheesh et al. [9] studied some properties of the AR (1) models. As Satheesh et al. [9] discussed, construct a first order autoregressive minification process with the following structure:

$X_{n}$

$=\left\{\begin{array}{l}\epsilon_{n} \text { with probability } \theta \\ \min \left(X_{n-1}, \epsilon_{n}\right) \text { with probability }(1-\theta), \quad 0 \leq \theta \leq 1,\end{array}\right.$

where $\left\{\epsilon_{n}\right\}$ is a sequences of i.i.d. random variables following a discrete uniform distribution with parameter $a$ independent of $\left\{X_{n-1}, X_{n-2}, \ldots\right\}$. Then the process is stationary and is 

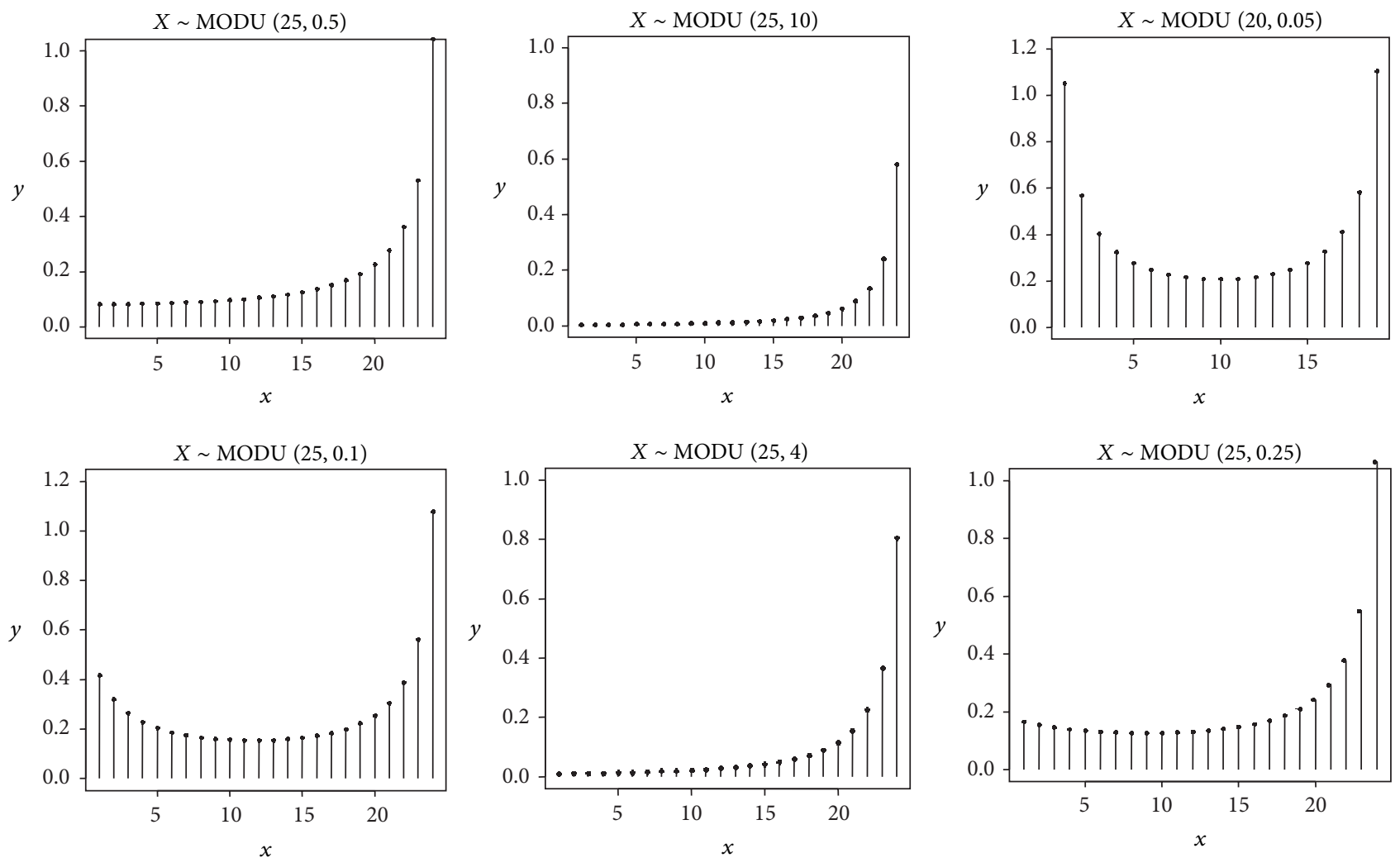

Figure 3: Hazard function $\gamma_{G}(x)$ of $X \sim \operatorname{MODU}(a, \theta)$.

TABLE 7: Mean of m.l.e. of $\theta$ from 10 repetitions. $(a=20, \widehat{a}=\max (x)=20$, sample size $=n)$.

\begin{tabular}{|c|c|c|c|c|}
\hline \multirow{3}{*}{$\theta$} & \multicolumn{4}{|c|}{$n$} \\
\hline & \multicolumn{2}{|c|}{2500} & \multicolumn{2}{|c|}{5000} \\
\hline & Mean $\widehat{\theta}$ & $\operatorname{SE}(\widehat{\theta})$ & Mean $\hat{\theta}$ & $\operatorname{SE}(\widehat{\theta})$ \\
\hline 0.1 & 0.1016693 & 0.1020 & 0.09995393 & 0.0020 \\
\hline 0.25 & 0.2487304 & 0.0020 & 0.2480182 & 0.0020 \\
\hline 0.5 & 0.5020383 & 0.0030 & 0.4920417 & 0.0030 \\
\hline 0.75 & 0.7512596 & 0.0023 & 0.7496541 & 0.0019 \\
\hline 0.9 & 0.9015150 & 0.0060 & 0.8997414 & 0.0043 \\
\hline 2 & 2.0007690 & 0.0191 & 1.9986450 & 0.0131 \\
\hline 5 & 4.9308800 & 0.0507 & 5.0252140 & 0.0362 \\
\hline 10 & 9.8801710 & 0.0900 & 9.9767230 & 0.0900 \\
\hline 50 & 51.069840 & 0.4321 & 100.04960 & 0.4006 \\
\hline 100 & 100.58670 & 1.3979 & 100.04960 & 0.4666 \\
\hline
\end{tabular}

marginally distributed with $\operatorname{MODU}(a, \theta)$ marginal distribution.

Theorem 12. In an $A R$ (1) process with structure (20) $\left\{Y_{i, n}\right\}$ is stationary Markovian with $\operatorname{MODU}(a, \theta)$ marginal if and only if $\left\{\epsilon_{n}\right\}$ is distributed as uniform with parameter $a, x=$ $1,2,3, \ldots, a$.

Proof. From the structure (20), it follows that

$$
\bar{F}_{X_{n}}(x)=\theta \bar{F}_{\epsilon_{n}}(x)+(1-\theta) \bar{F}_{X_{n-1}}(x) \bar{F}_{\epsilon_{n}}(x) .
$$

Under stationary equilibrium, this implies

$$
\bar{F}_{X}(x)=\frac{\theta \bar{F}_{\epsilon_{n}}(x)}{\left[1-(1-\theta) \bar{F}_{\epsilon_{n}}(x)\right]} .
$$

If we take $\bar{F}_{\epsilon_{n}}(x)=(a-x) / a$, then,

$\bar{F}_{X}(x)=\theta(a-x) /[a-(1-\theta)(a-x)]$, which is the survival function of $\operatorname{MODU}(a, \theta)$. We can also show that $\bar{F}_{\epsilon_{n}}(x)$ follows uniform distribution with parameter $a$. By retracing the steps we can easily show the converse. 
TABLE 8: Observed frequency of 50 students in 10 months from science and commerce classes.

\begin{tabular}{|c|c|c|c|c|c|c|c|c|c|c|c|c|c|c|c|c|c|c|c|c|}
\hline \multirow{2}{*}{ Month } & \multicolumn{20}{|c|}{$x$} \\
\hline & 1 & 2 & 3 & 4 & 5 & 6 & 7 & 8 & 9 & 10 & 11 & 12 & 13 & 14 & 15 & 16 & 17 & 18 & 19 & 20 \\
\hline May & 1 & 1 & 0 & 3 & 2 & 4 & 2 & 2 & 0 & 2 & 3 & 2 & 1 & 2 & 2 & 4 & 3 & 8 & 6 & 2 \\
\hline June & 0 & 2 & 1 & 2 & 4 & 1 & 1 & 1 & 2 & 4 & 2 & 2 & 1 & 3 & 2 & 4 & 5 & 5 & 3 & 5 \\
\hline July & 0 & 0 & 0 & 2 & 2 & 3 & 1 & 3 & 2 & 3 & 4 & 1 & 4 & 2 & 5 & 5 & 4 & 2 & 4 & 3 \\
\hline Aug & 2 & 1 & 3 & 1 & 1 & 1 & 1 & 2 & 3 & 1 & 1 & 4 & 4 & 2 & 1 & 6 & 5 & 3 & 3 & 5 \\
\hline Sep & 1 & 2 & 1 & 3 & 1 & 2 & 3 & 3 & 0 & 2 & 0 & 1 & 3 & 4 & 5 & 4 & 1 & 6 & 3 & 5 \\
\hline Oct & 1 & 0 & 2 & 0 & 3 & 0 & 2 & 3 & 0 & 6 & 2 & 4 & 3 & 1 & 4 & 4 & 2 & 8 & 2 & 3 \\
\hline Nov & 1 & 0 & 2 & 3 & 3 & 0 & 1 & 3 & 1 & 4 & 3 & 2 & 0 & 2 & 1 & 6 & 4 & 4 & 7 & 3 \\
\hline Dec & 1 & 3 & 0 & 1 & 3 & 3 & 2 & 0 & 4 & 0 & 2 & 1 & 2 & 5 & 2 & 4 & 4 & 5 & 3 & 5 \\
\hline Jan & 0 & 0 & 0 & 2 & 2 & 3 & 1 & 3 & 2 & 3 & 4 & 1 & 4 & 2 & 5 & 5 & 4 & 2 & 4 & 3 \\
\hline Feb & 2 & 1 & 3 & 1 & 1 & 1 & 0 & 2 & 3 & 1 & 1 & 4 & 4 & 2 & 2 & 6 & 5 & 3 & 3 & 5 \\
\hline
\end{tabular}

TABle 9: The m.l.e. of $\theta$ (sample size $=50$, m.l.e. of $a, \widehat{a}=20$ ).

\begin{tabular}{|c|c|c|c|c|c|c|c|c|c|c|}
\hline Month & May & June & July & Aug & Sept & Oct & Nov & Dec & Jan & Feb \\
\hline m.l.e. $\widehat{\theta}$ & 2.0347 & 2.0364 & 1.9247 & 2.0432 & 2.0347 & 1.9368 & 2.0743 & 1.9995 & 2.0039 & 1.995 \\
\hline
\end{tabular}

\section{Maximum Likelihood Estimates (m.l.e.) for the Parameters of $\operatorname{MODU}(a, \theta)$}

Let $x_{1}, x_{2}, \ldots, x_{n}$ be independent random samples from $\operatorname{MODU}(a, \theta)$. Then, from the likelihood function of the distribution, we can write

$$
\begin{gathered}
\left(\frac{n}{\theta}\right)-\sum \frac{\left(a-x_{i}\right)}{\left[a \theta+(1-\theta) x_{i}\right]} \\
\quad-\sum \frac{\left(a-x_{i}+1\right)}{\left[a \theta+(1-\theta)\left(x_{i}-1\right)\right]}=0, \\
\left(\frac{n}{a}\right)-\theta \sum \frac{1}{\left[a \theta+(1-\theta) x_{i}\right]} \\
-\theta \sum \frac{1}{\left[a \theta+(1-\theta)\left(x_{i}-1\right)\right]}=0 .
\end{gathered}
$$

We calculated the m.l.e. of $a$ and $\theta$ numerically (using Mathematica) as the solution of these two nonlinear equations. But, here, the maximum of the range of observation is $a$. So m.l.e. of the parameter $a(\widehat{a})$ is the largest value of the observation. Then, from (23), we can find m.l.e. of $\theta$.

Algorithm 13. Consider the following.

(1) Through simulation, 2500 random samples were generated from inverse function of distribution function $G(x)$ for some given value of the parameters $\theta$ and $a$.

(2) Then we iterate the m.l.e. for the parameters from the equations (23) and (24) (Table 6).

(3) For accuracy, we repeat the same calculation ten times with same values of $\theta$ and $a$.

(4) The mean and the standard error (SE) of these estimates are calculated.
TABLE 10: Mean m.l.e. of $\theta$ and mean Standard Error (SE) of $\theta$ for the samples of 10 months.

\begin{tabular}{lccc}
\hline Sample size & $\widehat{a}=\max (x)$ & Mean $\widehat{\theta}$ & $\operatorname{SE}(\widehat{\theta})$ \\
\hline 50 & 20 & 2.00832 & 0.04486918 \\
\hline
\end{tabular}

TABLE 11: Goodness of fit of uniform distribution with $\alpha=0.05$.

\begin{tabular}{lcccc}
\hline & May & August & October & February \\
\hline m.l.e. of $\theta=\hat{\theta}$ & 2.0347 & 2.0432 & 1.9368 & 1.995 \\
$\chi^{2}$ statistics & 14.800 & 9.4000 & 13.200 & 10.400 \\
$P$ value & 0.0632 & 0.3096 & 0.1052 & 0.2381 \\
\hline
\end{tabular}

TABle 12: Mean, median, and mode of the data in May, August, October, and February.

\begin{tabular}{cccccc}
\hline$a$ & Month & m.l.e. of $\theta=\hat{\theta}$ & Mean & Median & Mode \\
\hline \multirow{4}{*}{20} & May & 2.0347 & 12.80 & 14 & 18 \\
& August & 2.0432 & 12.30 & 13 & 16 \\
& October & 1.9368 & 12.86 & 13 & 18 \\
& February & 1.9950 & 12.64 & 14 & 16 \\
\hline
\end{tabular}

TABLE 13: Goodness of fit of MODU distribution with $\alpha=0.05$.

\begin{tabular}{lcccc}
\hline & May & August & October & February \\
\hline m.l.e. of $\theta=\hat{\theta}$ & 2.0347 & 2.04320 & 1.93680 & 1.99500 \\
$\chi^{2}$ statistics & 9.4668 & 6.45238 & 1.80953 & 6.95238 \\
$P$ value & 0.1489 & 0.37446 & 0.93640 & 0.325270 \\
\hline
\end{tabular}

(5) Then it is calculated with different values of the parameter $\theta$ and the same is found with an increase in sample size 5000 also.

(6) The mean estimated values for the parameters are tabulated (Table 7). 
TABLE 14: Observed frequency of 50 students in 10 months from the language classes.

\begin{tabular}{lcccccccccccccccccccccc}
\hline Month & & & & & & & & & & & & & & & & & & \\
& 1 & 2 & 3 & 4 & 5 & 6 & 7 & 8 & 9 & 10 & 11 & 12 & 13 & 14 & 15 & 16 & 17 & 18 & 19 & 20 \\
\hline May & 5 & 5 & 7 & 5 & 5 & 1 & 1 & 4 & 1 & 5 & 2 & 1 & 0 & 1 & 1 & 2 & 1 & 1 & 1 & 1 \\
June & 3 & 9 & 7 & 4 & 2 & 4 & 3 & 1 & 3 & 1 & 1 & 1 & 1 & 2 & 3 & 0 & 1 & 0 & 1 & 3 \\
July & 7 & 4 & 5 & 5 & 2 & 3 & 6 & 0 & 4 & 2 & 2 & 1 & 2 & 1 & 0 & 2 & 1 & 1 & 1 & 1 \\
Aug & 10 & 4 & 5 & 2 & 1 & 4 & 2 & 2 & 4 & 1 & 0 & 1 & 0 & 2 & 3 & 4 & 1 & 0 & 3 & 1 \\
Sep & 7 & 3 & 10 & 2 & 3 & 1 & 5 & 3 & 3 & 1 & 2 & 1 & 3 & 1 & 1 & 2 & 0 & 1 & 1 & 0 \\
Oct & 8 & 4 & 4 & 3 & 5 & 3 & 2 & 4 & 1 & 4 & 3 & 0 & 3 & 1 & 1 & 0 & 1 & 2 & 0 & 1 \\
Nov & 9 & 5 & 7 & 0 & 3 & 2 & 2 & 4 & 1 & 2 & 2 & 2 & 2 & 1 & 2 & 2 & 0 & 3 & 1 & 0 \\
Dec & 8 & 4 & 3 & 5 & 7 & 4 & 2 & 5 & 0 & 0 & 3 & 0 & 1 & 1 & 1 & 1 & 4 & 1 & 0 & 0 \\
Jan & 6 & 2 & 7 & 3 & 4 & 1 & 7 & 5 & 3 & 1 & 2 & 1 & 2 & 1 & 1 & 3 & 0 & 0 & 0 & 1 \\
Feb & 5 & 6 & 7 & 6 & 5 & 2 & 2 & 0 & 3 & 1 & 0 & 2 & 2 & 3 & 1 & 2 & 2 & 1 & 0 & 0 \\
\hline
\end{tabular}

TABLE 15: The m.l.e. of $\theta$ and SE of $\theta$ (sample size $=50$, m.l.e. of $a, \widehat{a}=\max (x)=20$ ).

\begin{tabular}{|c|c|c|c|c|c|c|c|c|c|c|}
\hline Month & May & June & July & Aug & Sept & Oct & Nov & Dec & Jan & Feb \\
\hline m.l.e. $\hat{\theta}$ & 0.3511 & 0.3544 & 0.3536 & 0.3802 & 0.3619 & 0.3522 & 0.3720 & 0.3707 & 0.3745 & 0.3594 \\
\hline
\end{tabular}

It is clear that the SE of m.l.e. of the parameters " $a$ " and $\theta$ is decreasing with increase in sample size.

\section{An Application of MODU}

Example 14. The data was collected from the daily attendance register of the science and commerce (nonlanguage) supplementary class (20 working days in a month) of a higher secondary school in Palakkad district, Kerala. We took a sample of 50 students (out of 360) and let $X$ be the number of days these students attended in the class for the whole year 2012-2013 (an academic year is 10 months) (see Tables 8, 9, and 10).

We arbitrarily fix 4 months, and the m.l.e.s are computed. Initially we fit discrete uniform to the data (see Tables 11 and 12).

Since mode $>$ median $>$ mean, the data exhibits negative skewness with m.l.e. of $\theta>1$ and it is observed that the distribution is unimodal; hence, MODU distribution is supposed to give a better fit than uniform distribution (see Table 13).

Result. From the $P$ values, it is seen that MODU distribution is better fit than uniform distribution.

Example 15. The data was collected from the daily attendance register of the supplementary language class (20 working days in a month) from the same higher secondary school in Palakkad district, Kerala. We took a sample of 50 students and let $X$ be the number of days these students attended in the same for the whole year 2012-2013 (an academic year is 10 months) (see Tables 14, 15, and 16).

We arbitrarily fix 3 months, and the m.l.e.s are computed. Fit discrete uniform to the above data. We have the results as shown in Tables 17 and 18.
TABLE 16: Mean m.l.e. of $\theta$ and mean SE of $\theta$ for the samples of 10 months.

\begin{tabular}{lccc}
\hline Sample size & $\widehat{a}=\max (x)$ & Mean $\widehat{\theta}$ & SE $(\widehat{\theta})$ \\
\hline 50 & 20 & 0.3630059 & 0.0033407 \\
\hline
\end{tabular}

TABLE 17: Goodness of fit of uniform distribution with $\alpha=0.05$.

\begin{tabular}{lccc}
\hline & May & September & February \\
\hline m.l.e. of $\theta=\widehat{\theta}$ & 0.3511 & 0.3630 & 0.3594 \\
m.l.e. of $a=\widehat{a}$ & 20 & 19 & 18 \\
$\chi^{2}$ statistics & 23.60 & 25.20 & 35.60 \\
$P$ value & 0.0027 & 0.0014 & 0.00002 \\
\hline
\end{tabular}

TABLE 18: Mean median and mode of the data in May, September, and February.

\begin{tabular}{cccccc}
\hline$a$ & Month & m.l.e. of $\theta=\hat{\theta}$ & Mean & Median & Mode \\
\hline \multirow{2}{*}{20} & May & 0.3510 & 7.80 & 5 & 3 \\
& September & 0.3619 & 6.78 & 5 & 1 \\
& February & 0.3594 & 6.82 & 5 & 3 \\
\hline
\end{tabular}

TABLE 19: Goodness of fit of MODU distribution with $\alpha=0.05$.

\begin{tabular}{lccc}
\hline & May & September & February \\
\hline m.l.e. of $\theta=\widehat{\theta}$ & 0.3511 & 0.3630 & 0.3594 \\
$\widehat{a}$ & 20 & 19 & 18 \\
$\chi^{2}$ statistics & 9.80007 & 7.23453 & 7.79048 \\
$P$ value & 0.1333 & 0.2997 & 0.2538 \\
\hline
\end{tabular}

Since mode $<$ median $<$ mean, the data exhibits positive skewness with m.l.e. of $\theta<1$ and it is observed that the distribution is unimodal; hence, MODU distribution is supposed to give a better fit than uniform distribution (see Table 19). 
Result. From the $P$ values it is seen that, here, MODU distribution is a better fit than uniform distribution.

\section{Conclusions}

The p.m.f.s of MODU distribution $g(x, \theta)$ satisfies the relation $g(x, \theta)=g(a-x+1,1 / \theta), x=1,2, \ldots, a, \theta>0$. The failure rate of $\operatorname{MODU}(a, \theta)$ is IFR, when $\theta>(a-2 x) /(2 a-2 x)$, DFR, when $\theta<(a-2 x) /(2 a-2 x)$, and constant, when $\theta=(a-2 x) /(2 a-2 x)$. Like discrete uniform distribution, the probability function degenerates when a is large. In the case of $\operatorname{MODU}(a, \theta)$, the standard deviation is decreasing with the increasing value of $\theta$, when $\theta>1$, and is increasing with increasing value of $\theta$, when $\theta<1$. For $X \sim \operatorname{MODU}(a, \theta)$ and $X \sim \operatorname{MODU}(a, 1 / \theta)$, the $\operatorname{sd}(X)$ is equal in both cases. It is also observed that entropy $(a, \theta)=\operatorname{entropy}(a, 1 / \theta)$. The MODU distribution is positively skewed, when $\theta<1$, since, here, mode $<$ median $<$ mean and the distribution is negatively skewed, when $\theta>1$, since then mode $>$ median $>$ mean. It is to be noted that the distribution is unimodal; that is, when $\theta<1$, the mode $=1$ and, when $\theta>1$, mode $=a$. An application of MODU distribution is also discussed.

\section{Conflict of Interests}

The authors declare that there is no conflict of interests regarding the publication of this paper.

\section{References}

[1] A. W. Marshall and I. Olkin, "A new method for adding a parameter to a family of distributions with application to the exponential and Weibull families," Biometrika, vol. 84, no. 3, pp. 641-652, 1997.

[2] K. K. Jose and T. Alice, Marshall-Olkin Family of DistributionsApplications in Time Series Modeling and Reliability, JC Publications, 2005.

[3] K. K. Jose and E. Krishna, Marshall-Olkin Extended Uniform Distribution, ProbStat Forum, 2011.

[4] S. Satheesh and N. U. Nair, "A note on maximum and minimum stability of certain distributions," Calcutta Statistical Association Bulletin, vol. 53, pp. 249-252, 2002.

[5] S. Satheesh and N. U. Nair, "On the stability of geometric extremes," International Indian Statistical Association, vol. 42, pp. 99-109, 2004.

[6] S. Satheesh, E. Sandhya, and N. U. Nair, "Stability of random sums," Stochastic Modelling and Applied Probability, vol. 5, pp. 17-26, 2002.

[7] B. C. Arnold and C. A. Robertson, "Autoregressive logistic processes," Journal of Applied Probability, vol. 26, no. 3, pp. 524$531,1989$.

[8] M. R. Chernick, "A limit theorem for the maximum of autoregressive processes with uniform marginal distributions," Annals of Probability, vol. 9, no. 1, pp. 145-149, 1981.

[9] S. Satheesh, E. Sandhya, and S. Sherly, "A generalization of stationary AR(1) schemes," Statistical Methods, vol. 8, no. 2, pp. 213-225, 2006. 


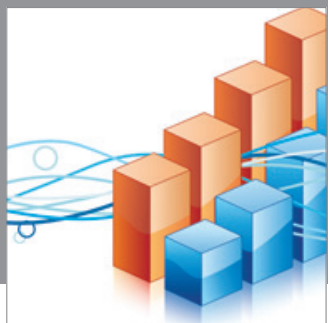

Advances in

Operations Research

mansans

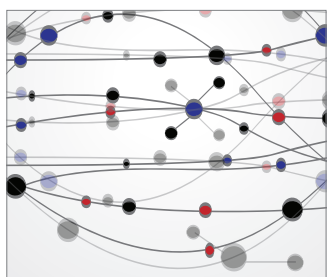

The Scientific World Journal
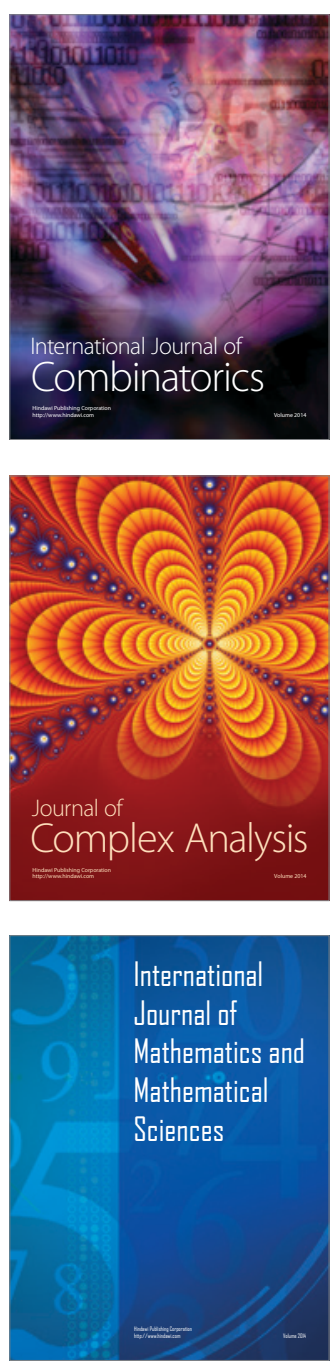
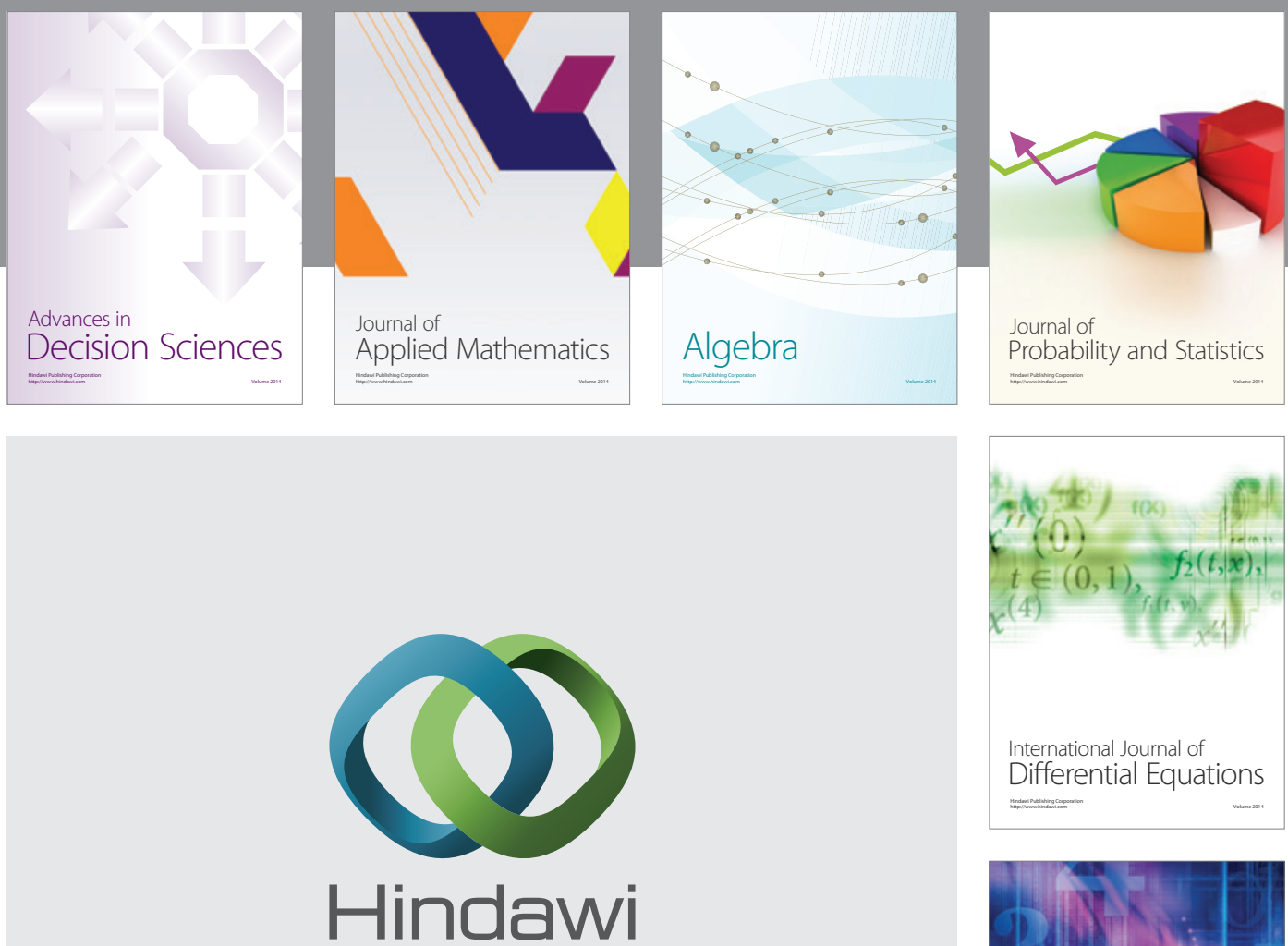

Submit your manuscripts at http://www.hindawi.com
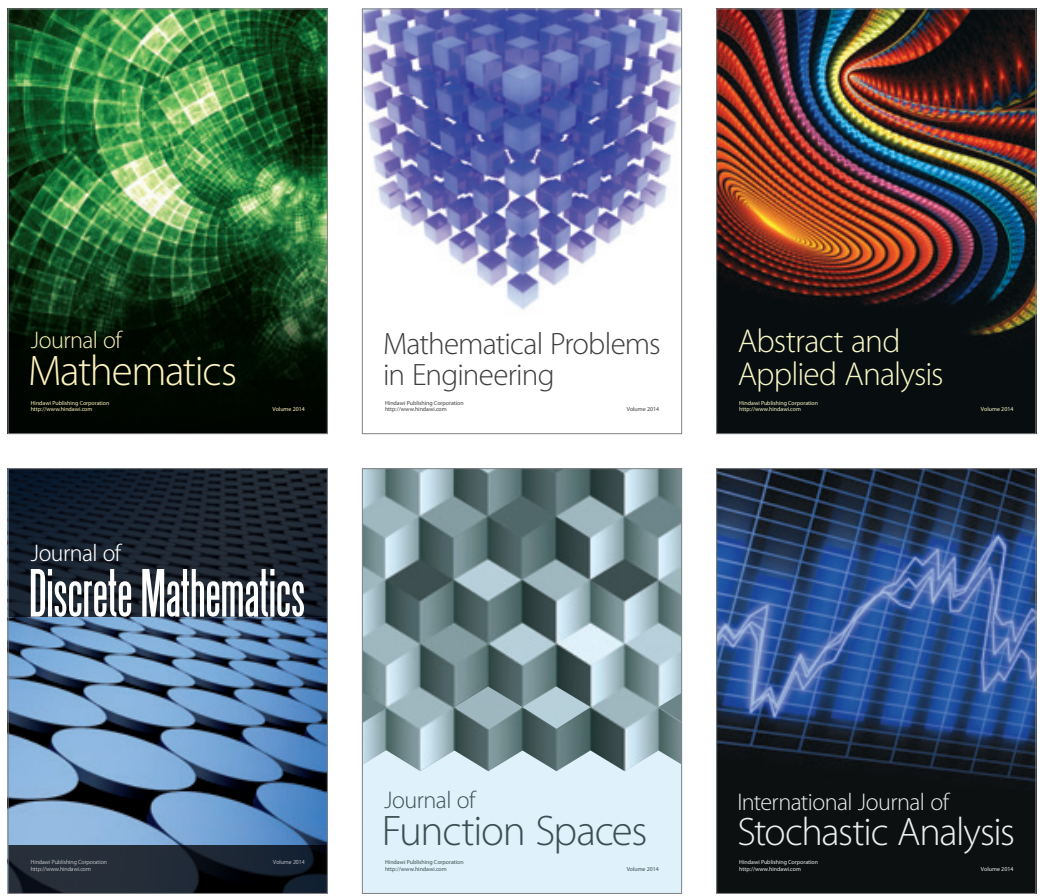

Journal of

Function Spaces

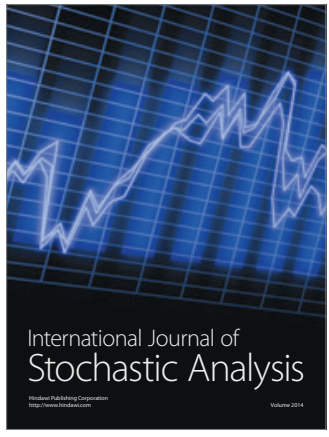

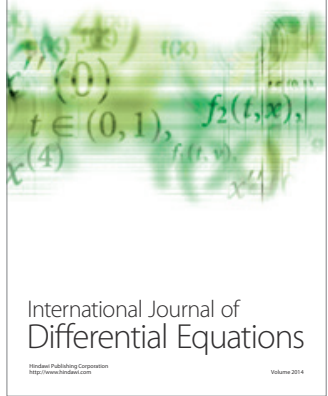
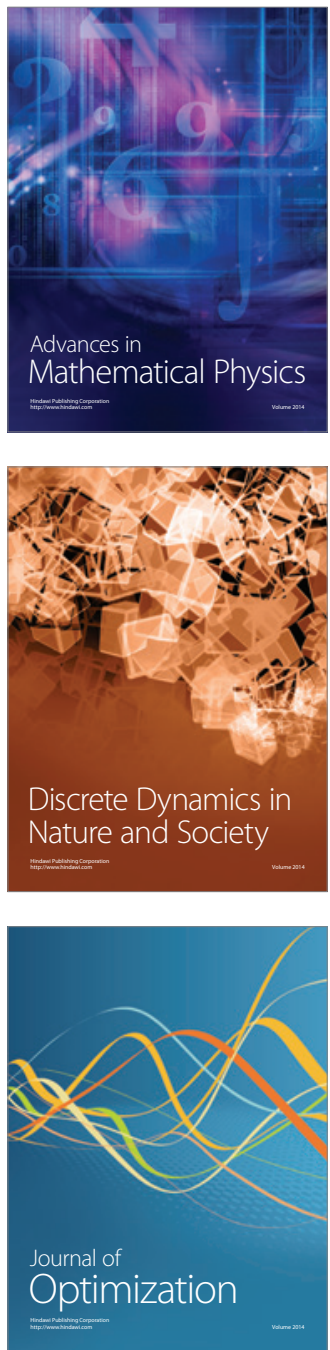